\title{
Multi-Phase Space Vector Pulse Width Modulation: Applications and Strategies.
}

\author{
Mario J. Duran ${ }^{1}$, F. Barrero ${ }^{2}$ and S. Toral ${ }^{2}$ \\ ${ }^{1}$ Electrical Engineering Department \\ School of Engineering, University of Seville \\ Camino de los descubrimientos S/N, 41092, Sevilla (Spain) \\ Tlf. 954 481281, email: mjduran@us.es \\ ${ }^{2}$ Electronic Engineering Department \\ School of Engineering, University of Seville \\ Camino de los descubrimientos S/N, 41092, Sevilla (Spain) \\ Tlf. 954 487368, email: fbarrero@esi.us.es, toral@esi.us.es
}

\begin{abstract}
Multi-phase machines and drives is a topic of growing relevance in recent years, and it presents many challenging issues that still need further research. This is the case of multi-phase space vector pulse width modulation (SVPWM), which shows not only more space vectors than the standard three-phase case, but also new subspaces where the space vectors are mapped. Different approaches have been recently followed, and the aim of this paper is to review and classify these methods. Comparative results are included to highlight the weak and strong points of the different methods. Finally some conclusions are extracted pointing out the problems that still need to be solved.
\end{abstract}

Keywords: Multi-phase systems, voltage source inverter, space vector modulation, pulse width modulation.

\section{Introduction}

The interest in multi-phase motor drives has increased in recent years due to several advantages when compared to three-phase drives [1-2]. Some of these advantages are known from the early days of the multiphase drives [3], although they have recently considered with high-level analysis [4]. These advantages are inherent to the own structure of the machine, and include less torque ripple, less acoustic noise and losses, reduced current per phase or increased reliability due to the additional number of phases [1-4]. These advantages make multi-phase drives suitable for high power/current applications such as EV/HEVs, traction or electric ship propulsion. Apart from these benefits, there are further possibilities due to the additional degrees of freedom existing in multi-phase machines. However, there is a common feature to all applications: a proper modulation technique needs to be designed to achieve high performance. Among different methods, space vector pulse width modulation (SVPWM) is one of the most popular choices due to the easy digital implementation and because of a better utilization of the available dc bus voltage [5]. However, the extension of SVPWM algorithms from the three to multi-phase systems is far from trivial. This is due to both increasing number of space vectors $\left(2^{\mathrm{n}}\right.$ being $n$ the number of phases) and subspaces where the vectors are simultaneously mapped $((n-1) / 2$ assuming $n$ is an odd number). If a $d-q$ subspace appears in a three-phase machine, additional $x-y$ subspaces appear in the general $n$-phase case. The vector space decomposition (VSD) from the phase variables into the different planes or subspaces is explained by Zhao and Lipo [6], and it helps to understand the high currents appearing in multi-phase machines due to the $x-y$ currents. Additionally, it is the basis for new applications such as torque enhancement in concentrated-winding machines, series connection of multi-phase machines or faulttolerant mode of operation. This paper makes a summary of these applications and classifies the existing SVPWM algorithms, indicating their validity for each application and the limitations of each approach.

The paper is structured so that sections 2 briefly summarizes the novel applications based on the additional degrees of freedom existing in multi-phase machines, while Section 3 includes details about the VSD approach, the modelling of the $n$-phase converter and the quality parameters usually considered for comparative purposes. Section 4 classifies the existing methods showing the differences and limitations of each one, and section 5 extracts the most relevant conclusions and aims to encourage researchers to improve the existing limitations for some particular applications. 


\section{Novel multi-phase drives applications.}

The additional degrees of freedom existing in multiphase machines allow designing new applications which can not be implemented in standard three-phase machines [1-2]. The three main applications are briefly summarized:

1. Torque enhancement by current harmonic injection: If the machine has distributed windings, the injection of current harmonics can lead to torque ripple or not produce any effect. However, if the machine has concentrated windings, the spatial harmonics interact with the current harmonics producing an airgap field. This additional field rotates at fundamental speed if the order of the current and spatial harmonics is the same. This fact leads to increase the torque density by injecting harmonics using the $x-y$ components [14]-[16]. The fundamental component is generated using the $d-q$ subspace, while the harmonics are injected using the $x-y$ components. A third harmonic can be injected in a five-phase machine, a third and a fifth harmonics in a seven-phase machine, and so on.

2. Disturbance-free mode of operation: If one or more phases need to be open-circuited in a three-phase machine, the rotating field can no longer be generated in the post-fault situation leading to torque ripple. Nevertheless, multiphase machines can operate after the loss of one or more phases if the current in the other phases is changed to compensate for the faulted phase [7-10]. However, when one phase is open-circuited, one degree of freedom is lost, and the $x-y$ components can no longer be set to zero. A restriction is included for each faulted phase and the generation of the rotating field needs to include, as in the previous case, $x-y$ components.

3. Independent control of machines supplied from a single inverter: multi-phase machines can be connected in series [11]-[13] or in parallel [12] while supplied from a single inverter. This application allows an independent control of the machines because each machine is controlled (with appropriate transposition of phases) using two degrees of freedom from a subspace (e.g. $d-q$ for one machine and $x-y$ components for another machine). The independency can be achieved because the different planes are mutually orthogonal.
The three applications have the common feature of requiring non-zero $x-y$ components. Although the usual case of sinusoidal output voltage has found successful SVPWM algorithms, it will be shown in the next sections that the complexity to find a proper SVPWM scheme for these applications is higher and a completely satisfactory solution has not still been obtained.

\section{Modelling of the $n$-phase inverter.}

Power circuit topology of an $n$-phase VSI is shown in Fig. 1. The inverter input dc voltage is treated as constant and its value is set to 1 p.u. later on for simulation purposes. The load is taken as starconnected with isolated neutral point so that the zerosequence cannot be excited and one therefore deals further on, for odd $n$, with an (n-1) dimensional space. The inverter leg voltages are referenced to the midpoint of the dc supply and notation for inverter leg and phase voltages is defined in Fig. 1. A switching function $S_{i} \in\{1,-1\}$ is defined for each inverter leg, $i=$ $1,2 \ldots n$. The leg voltages are then determined with

$V_{i}=0.5 V_{D C} S_{i}, \quad S_{i}= \pm 1, \quad i=1,2 \ldots . . n$

The inverter output phase-to-neutral voltages can be written as functions of inverter leg voltages,

$$
\begin{gathered}
v_{1}=\frac{n-1}{n} V_{1}-\frac{1}{n} \sum_{i=2}^{n} V_{i} \\
v_{2}=\frac{n-1}{n} V_{2}-\frac{1}{n} \sum_{i=1, i \neq 2}^{n} V_{i} \\
\vdots \\
v_{n}=\frac{n-1}{n} V_{n}-\frac{1}{n} \sum_{i=1}^{n-1} V_{n}
\end{gathered}
$$

Since the $(n-1)$ dimensional space can be decomposed into (n-1)/2 two-dimensional sub-spaces according to the VSD approach, phase voltage space vectors can be defined for each sub-space as

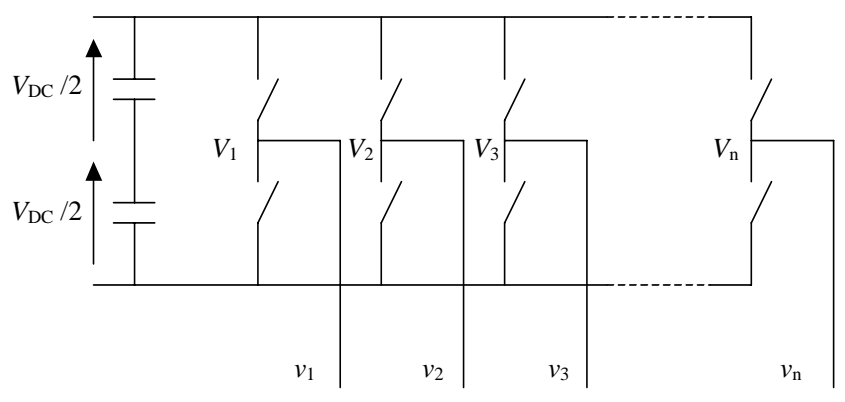

Fig. 1. An $n$-phase two-level voltage source inverter. 
$\underline{v}_{d q}=v_{d}+j v_{q}=\frac{2}{n} \sum_{i=1}^{n} v_{i} \underline{a}^{i-1}$

$\underline{v}_{x y}^{1}=v_{x}^{1}+j v_{y}^{1}=\frac{2}{n} \sum_{i=1}^{n} v_{i} \underline{a}^{(i-1) \cdot 2}$

$\underline{v}_{x y}^{2}=v_{x}^{2}+j v_{y}^{2}=\frac{2}{n} \sum_{i=1}^{n} v_{i} \underline{a}^{(i-1) \cdot 3}$

$\underline{v}_{x y}^{(n-3) / 2}=v_{x}^{(n-3) / 2}+j v_{y}^{(n-3) / 2}=\frac{2}{n} \sum_{i=1}^{n} v_{i} \underline{a}^{(i-1) \cdot(n-1) / 2}$

where $\underline{a}=\exp (j 2 \pi / n)$.

The first subspace is termed $d-q$, and the currents flowing in this plane are responsible for the creation of the rotating field in the airgap of the machines. On the other hand, $x-y$ components are responsible for the additional losses in distributed-winding machines, since the currents flowing in this subspace cancel each other and do not link the rotor side. Consequently, unless space harmonics appear, $x-y$ components are not electromechanically related. The $2^{\mathrm{n}}$ VSI space vectors including all commutation possibilities are simultaneously mapped into all subspaces. In the fivephase case, there are 2 zero vectors and 30 active vectors that appear in both $d-q$ and $x-y$ planes, as shown in Fig. 2. A multi-dimensional vector can be defined, and the k-th vector can be expressed as:

$\underline{v}_{k}=\left[\begin{array}{lllllll}v_{k 1} & v_{k 2} & v_{k 3} & v_{k 4} & \ldots & v_{k(n-2)} & v_{k(n-1)}\end{array}\right]$

where indices 1,2 indicate $d-q$ components while indices $3,4, \ldots(n-2),(n-1)$ stand for $x-y$ components. In order to generate the reference vector with the proper application of the VSI active space vectors, $k=n-1$ active vectors need to be selected and the following $(n-1) \times(n-1)$ matrix can be written:

$$
\underline{M}=\left[\begin{array}{cccc}
v_{11} & v_{21} & \cdots & v_{(n-1) 1} \\
v_{12} & v_{22} & \cdots & v_{(n-1) 2} \\
\vdots & \vdots & \ddots & \vdots \\
v_{1(n-1)} & v_{2(n-1)} & \cdots & v_{(n-1)(n-1)}
\end{array}\right]
$$

The reference voltage vector can then be created on average during one switching period by applying the $(n-1)$ selected active space vectors for certain time intervals

$\left[\begin{array}{c}v_{d}^{*} \\ v_{q}^{*} \\ \vdots \\ v_{x}^{(n-3) / 2} \\ v_{y}^{(n-3) / 2}\end{array}\right] T_{s}=\left[\begin{array}{cccc}v_{11} & v_{21} & \cdots & v_{(n-1) 1} \\ v_{12} & v_{22} & \cdots & v_{(n-1) 2} \\ \vdots & \vdots & \ddots & \vdots \\ v_{1(n-1)} & v_{2(n-1)} & \cdots & v_{(n-1)(n-1)}\end{array}\right]\left[\begin{array}{c}t_{1} \\ t_{2} \\ \vdots \\ t_{(n-1)}\end{array}\right]$
The required times of application for the selected $(n-1)$ active vectors follow then in the form

$$
\underline{t}=\underline{M}^{-1} \underline{v}^{*} T_{s}
$$

where $T_{s}$ is the switching period and the time of application of zero vectors is

$t_{0}=T_{s}-\sum_{k=1}^{n-1} t_{k}$

It is assumed that the selected active space vectors are pre-ordered so that the switching stress is minimised and that symmetrical SVPWM is applied.

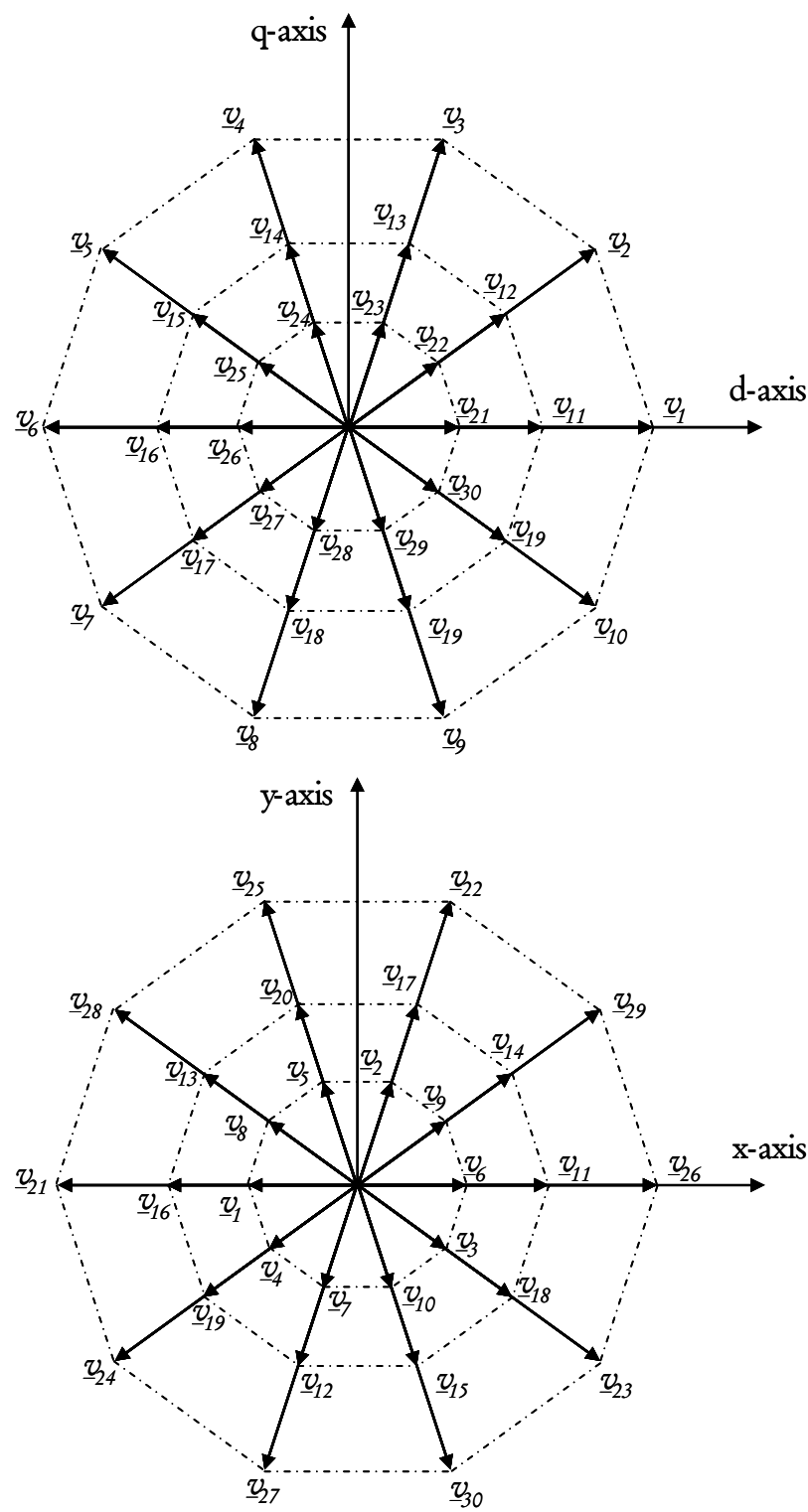

Fig. 2. Phase voltage space vectors of a five-phase VSI in $d-q$ and $x-y$ planes. 
Following this approach, the SVPWM for multi-phase drives can be achieved, but it has been assumed that the active space vectors have been already selected. If these vectors are not the adjacent in $d-q$ subspace, the selected vectors need to satisfy some conditions to assure that the reference can be synthesized. The validity conditions are [20], [26], [27]:

1) The selected $(n-1)$ vectors must be linearly independent. This condition is satisfied if the determinant of the square matrix $\underline{M}$ of (6) differs from zero.

2) The reference vector $\underline{v}^{*}$ must be positioned within boundaries of the $(n-1)$ dimensional space formed by the selected vectors. This ensures that the reference voltage can be built using the selected set of active vectors. The easiest way to check whether this condition is satisfied is by verifying that all the calculated application times for selected space vectors in (8) are positive. Additionally, the zero vector application time in (9) must satisfy $t_{0} \geq 0$.

These two conditions will be referred to as validity conditions from now on. If these conditions are satisfied, the reference vector can be built by applying the selected set of $(n-1)$ active vectors of (5). However, the reference voltage can be achieved in the mean during the switching period with different selections of the VSI space vectors, which will yield different inverter performance.

\section{Multi-phase SVPWM strategies.}

The procedure outlined in the previous section is a generalization of [6] and is based on a general analysis of the $n$-phase case as in [18]-[19]. The validity conditions were firstly established in [20] and the selection of the (n-1) active vectors has been done from several perspectives that are summarized in this section. Some of the methods are valid for sinusoidal output voltage generation while others are generally applicable to any case. The methods for space vector selection can be divided into those that select the vector based on the proximity of active vectors in a subspace, and those that are based on the proximity of active vectors in a multidimensional space. The former will be referred as VSD methods and the latter as multi-dimensional methods. Among the first group, the following approaches have been followed:

a) Selection of $(n-1) / 2$ vectors considering the $d-q$ plane only.

This method was proposed in [21]-[22] for the generation of sinusoidal output voltage of a five-phase drive. It is the natural extension of three-phase
SVPWM since it considers different sectors in the $d-q$ plane and selects the adjacent vectors in each sector. These vectors are the large ones achieving maximum modulation index for sinusoidal output voltage. However, the selections of $(n-1) / 2$ vectors provides $(n$ 1)/2 equations that cannot satisfy the $x-y$ components. Consequently, $x-y$ components appear that lead to high stator currents that are limited only by stator leakage inductance and resistance. These currents generate losses and do not contribute to the torque generation [6], leading to inadequate drive performance.

\section{b) Selection of (n-1) vectors considering the $d-q$ plane only.}

Because of the high $x-y$ currents appearing in multiphase machines, it was stated that it is necessary $(n-1)$ space vectors to satisfy not only $\mathrm{d}-\mathrm{q}$ reference components, but also $x-y$ reference components [20]. This approach has been followed in several studies for different number of phases. It has been most frequently considered in five-phase drives were the medium and large VSI space vectors are selected in each sector [23], [28]-[29] and they are applied with percentages of 61.8 $\%$ and $38.2 \%$ respectively to cancel $x-y$ components. The maximum peak fundamental of the output phase voltage (assuming dc voltage of 1 p.u.) using this approach is 0.5257 p.u. for a five-phase VSI if the medium and large adjacent vectors in each sector of the $d-q$ plane are selected (e.g. a $d$ - $q$ reference space vector in the first sector for a five-phase VSI would be created from vectors 1, 2, 11 and 12 of Fig. 2). The transition from this scheme to the previous one with $(n-1) / 2$ vectors has been explored in [23] achieving an extended modulation index at the expenses of increasing harmonic content. Basically, the percentage of application of large vectors is increased from $38.2 \%$ to $100 \%$ when the maximum modulation index is achieved. This approach has been followed for the generation of fundamental and third harmonic in torque enhancement applications [28]-[29], but the harmonic content is limited if the validity conditions are to be satisfied. The application of $(n-1)$ vectors has also been considered for seven-phase drives [17] and for the general $n$-phase case [18]-[19]. However, from the vector selection point of view the followed approach is similar.

c) Selection of active vectors considering the $d-q$ and $x-y$ planes. The case of arbitrary references in the two planes is considered here. Since only $(n-1)$ vectors are necessary to build the reference $(n-1) / 2$ vectors, it is theoretically possible to chose these vectors by considering one reference vector in each 
sub-space ( $d-q$ and $x-y)$ and then to select two vectors (for five-phase VSI) from each sub-space adjacent to the corresponding reference. This possibility was suggested in [26], but it has been verified that the simple solution of selecting large vectors in both $d-q$ and $x-y$ planes (e.g. for a fivephase VSI with a reference $d-q$ space vector in the first sector and a $x-y$ reference vector in the third sector, the selected vectors would be 1,2 , and 22 , 25 , respectively) does not satisfy the second validity condition. While in principle this approach to active vector selection may be feasible, it requires further work.

d) Selection of $(n-1) \times(n-1) / 2$ vectors. In this case $(n-1)$ vectors are selected in each plane (the total number of planes is $(n-1) / 2)$ to synthesize the reference component in its own plane. In the five-phase case, four active vectors are selected to build the $d-q$ reference while simultaneously eliminating $x-y$ components and another four vectors are selected to build the $x-y$ reference while simultaneously eliminating the $d-q$ components. For a five-phase VSI with a $d-q$ space vector in the first sector and a $x-y$ vector in the third sector, the selected vectors would be $1,2,11,12$, and $17,20,22,25$, respectively. This approach, based on decoupling of the different sub-spaces, is followed in [24] and it synthesizes both $d-q$ and $x-y$ references. However, the implementation method described in [24] uses two successive switching periods to alternately synthesize the two reference vectors, so that the maximum achievable fundamental is automatically restricted to half of the value that would have been achievable with given dc link voltage in the case of, say, reference in only $d-q$ plane.

Among the second group two approaches can be followed:

e) Selection of (n-1) vectors by defining spatial sectors in the (n-1) dimensional space.

This solution was discarded in [25] due to the huge amount of sectors and the authors' investigation confirms that application of this approach even for the simplest case of a five-phase VSI is unlikely.

f) On-line selection of (n-1) vectors using a multidimensional approach not based on the sector belonging criterion.

This is the approach has been detailed in [20], [26][27] for the three main applications were non-zero $x$ $y$ components appear. The approach follows the idea suggested in [25] and proposes an improved criterion that converges with the solutions exposed in [17]-[19], [23], [28]-[29] for sinusoidal output voltage. However, the method proves to be general for any reference (including non-zero $x-y$ components) and number of phase. From this perspective, the idea of sectors is lost. As an example, when the angle of the $d-q$ reference space vector is $3.6^{\circ}$ and the angle of the $x-y$ reference space vector is $93.6^{\circ}$, the selected vectors are 20,22 , 2 and 13. It can be observed that there is a combination of vectors with different lengths (one large, one small and two medium vectors) and the selected vectors are not necessarily adjacent in any of the planes. This result is not intuitive considering the problem from the VSD point of view, but it is a straightforward result from the multi-dimensional approach. Three main disadvantages can be associated with this approach: the algorithm is computationally intense, the execution time is not constant and the switching frequency is slightly increased compared to sinusoidal case. On the other hand it improves the performance of [24] because it shows a lower switching frequency and flexibility to operate with different references for the $d-q$ and $x-y$ planes. As an example, Fig. 3 shows the waveform and spectrum of phase 2 voltage for the disturbancefree operation when phase 1 is open-circuited. It can be observed that the fundamental is achieved while the unwanted harmonics practically do not exist.
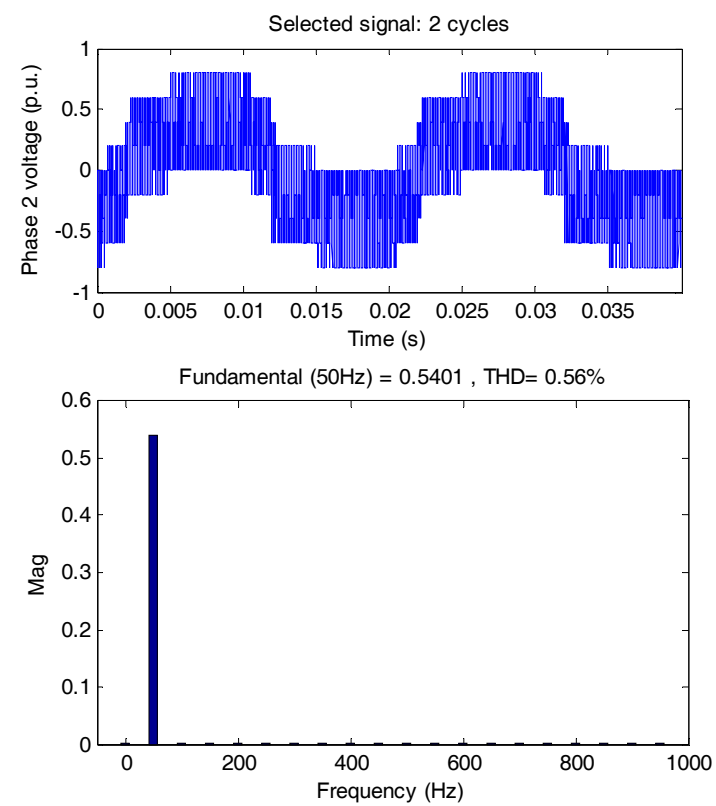

Fig. 3. Output phase 2 voltage waveform and associated spectrum when phase 1 is open and the reference $d-q$ voltage is set to 0.368 p.u. 
The main points to be considered in the comparative analysis are:

All the different options are valid for sinusoidal output voltage generation while only options d) and f) are valid for the general case when non-zero $x-y$ components appear.

$>$ Options a) to d) inherently satisfy the validity conditions and show minimum switching frequency, while option f) needs to verify the validity conditions in real time and the switching frequency is slightly increased for non-sinusoidal output voltage generation.

$>$ The maximum modulation index is achieved with option a) and b) for the sinusoidal output voltage case, while option f) obtains the better dc bus utilization with absence of unwanted harmonics.

However, there are some remaining points to be improved if one wishes to combine the advantages from the different methods:

A general algorithm with minimum switching frequency must be achieved.

$>$ It needs to be devised a way to use a general method with lower computational cost.

$>$ An extension of the general case to be able to increase the modulation index, even at the expenses of harmonic appearance, should be designed.

\section{Conclusions.}

The main applications of multi-phase drives can be divided into those with sinusoidal output voltage requirements and those with non-sinusoidal output voltage generation. The SVPWM for the first group has been recently achieved with success using a sectorbased approach that leads to minimum switching frequency, simple algorithms and maximum modulation index. However, the second group of applications requires algorithms with increased complexity. It has been shown that the methods proposed for sinusoidal output voltage are no longer valid in the general case since some validity conditions need to be satisfied. There are two approaches for this second group of applications: VSD-based and multidimensional approaches. The current VSD-based methods present some limitations regarding switching frequency and unequal share of the dc bus voltage. On the other hand, multi-dimensional methods shows a good performance but at the expenses of algorithms with increased switching frequency and high computational cost. All in all, the current SVPWM methods are optimum for sinusoidal applications but still present some disadvantages for general nonsinusoidal applications.

\section{References.}

[1] Levi, E., Bojoi, R., Profumo, F., Toliyat, H.A., and Williamson, S., "Multiphase induction motor drives - a technology status review'” IEE Elec. Power appl., 2007, (in press).

[2] Singh, G.K., "Multi-phase induction machine drive research a survey”, Electric Power Systems Research, 61, pp. 139-147, 2002.

[3] E. E. Ward and H. Härer, "Preliminary investigation of an inverter-fed 5-phase induction motor," Proc. IEE, Vol. 116, No. 6, pp. 980-984, 1969

[4] Williamson, S., and Smith, A.C., "Pulsating torque and losses in multiphase induction machines", IEEE Trans. on Industry Applications, vol. 39, no. 4, pp. 986-993, 2003.

[5] G.D. Holmes, T.A. Lipo, "Pulse Width Modulation for Power Converters - Principles and Practice,” IEEE Press Series on Power Engineering, John Wiley and Sons, Piscataway, NJ, USA, 2003.

[6] Y. Zhao, T.A. Lipo, "Space vector PWM control of dual three-phase induction machine using vector space decomposition,” IEEE Trans. on Industry Applications, vol. 31, no. 5, pp. 1100-1109, 1995.

[7] J.R.Fu, T.A.Lipo, "Disturbance free operation of a multiphase current regulated motor drive with an opened phase”, IEEE Trans. on Industry Applications, vol. 30, no. 5, 1994, pp. 1267-1274.

[8] C.B. Jacobina, I.S. Freitas, T.M. Oliveira, E.R.C. da Silva, A.M.N. Lima, "Fault tolerant control of five-phase AC motor drive", in Proceedings 35th Annual IEEE Power Electronics Specialist Conference, PESC, Aachen, Germany, 2004, pp. 3486-3492.

[9] L. Parsa, H.A. Toliyat, "Fault-tolerant five-phase permanent magnet motor drives", in Proceedings of the IEEE Ind. Appl. Soc. Annual Meeting IAS, Seattle, USA, 2004, pp. 1048-1054.

[10] Durán, M.J., Barrero, F., Toral, S., Gallardo, S., "Funcionamiento de máquinas polifásicas ante la pérdida de una o varias fases”, SAAEI, Gíjón, 2006.

[11] E. Levi, M. Jones, S. Vukosavic and H.A. Toliyat, “A novel concept of a multiphase, multi-motor vector controlled drive system supplied from a single voltage source inverter," IEEE Trans. on Power Electronics, vol. 19, no. 2, 2004, pp. 320335.

[12] Jones, M., Levi, E., Vukosavic, S., “Independent control of two five-phase induction machines connected in parallel to a single inverter supply”, in Proceedings IEEE 32nd Annual Conference of the IEEE Industrial Electronics Society. IECON, Paris, France, 2006.

[13] Duran, M.J., Levi, E., and Jones, M., '’Independent vector control of asymmetrical nine-phase machines by means of series connection', Proc. IEEE Int. Electric Machines and Drives Conf. IEMDC, San Antonio, TX, pp. 167-173, 2005.

[14] Toliyat, H.A., Waikar, S.P., and Lipo, T.A., 'Analysis of simulation of five-phase synchronous reluctance machines including third harmonic airgap MMF', IEEE Trans. on Industry Applications, vol. 34, no. 2, pp. 332-339, 1998. 
[15] Xu, H., Toliyat, H.A., and Petersen, L.J., 'Rotor field oriented control of a five-phase induction motor with the combined fundamental and third harmonic currents', Proc. IEEE Applied Power Electronics Conference APEC, Anaheim, CA, pp. 392398, 2001.

[16] Xu, H., Toliyat, H.A., and Petersen, L.J., 'Five-phase induction motor drives with DSP-based control system', IEEE Trans. on Power Electronics, vol. 17, no. 2, pp. 524-533, 2002.

[17] G.Grandi, G.Serra, A.Tani, "Space vector modulation of a seven-phase voltage source inverter", in Proc. Int. Symp. on Power Electronics, Electrical Drives, Automation and Motion SPEEDAM, Taormina, Italy, 2006, pp. S8-6-S8-13.

[18] J.W. Kelly, E.G. Strangas, J.M. Miller, "Multi-phase space vector pulse width modulation," IEEE Trans. on Energy Conversion, vol. 18, no. 2, 2003, pp. 259-264.

[19] G.Grandi, G.Serra, A.Tani, "General analysis of multi-phase systems based on space vector approach", in Proc. EPEPEMC Conf., Portoroz, Slovenia, 2006, pp. 834-840.

[20] Durán, M.J., Levi, E. "Multi-dimensional approach to multiphase space vector modulation" in Proceedings IEEE 32nd Annual Conference of the IEEE Industrial Electronics Society. IECON, Paris, France, 2006.

[21] Toliyat, H.A., Shi, R., Xu, H., "A DSP-based vector control of five-phase synchronous reluctance motor", Proc. IEEE IAS Annual Meeting, Rome, Italy, 2000, CD-ROM paper No. 40_05.

[22] Toliyat, H.A., Xu, H., "DSP-based direct torque control (DTC) for five-phase induction machines" Proc. Int. Power electronics Conf. IPEC, Tokyo, Japan, 2000, p. 1195-1200.
[23] A. Iqbal, E. Levi, "Space vector PWM techniques for sinusoidal output voltage generation with a five-phase voltage source inverter," Electric Power Components and Systems, vol. 34 no. 2, 2006, pp. 119-140.

[24] A.Iqbal, E.Levi, "Space vector PWM for a five-phase VSI supplying two five-phase series-connected machines", in Proc. Int. Power Electronics and Motion Control Conf. EPEPEMC, Portoroz, Slovenia, 2006, pp. 222-227.

[25] Gataric, S., 'A polyphase Cartesian vector approach to control of polyphase AC machines,' in Proceedings of the IEEE Ind. Appl. Soc. Annual Meeting IAS, Rome, Italy, 2000, CD-ROM paper no. 38_02.

[26] Durán, M.J., Levi, E., "Multi-dimensional space vector pulse width modulation schme for five-phase series-connected twomotor drive", IEEE Int. Elec. Machines and Drives Conf. IEMDC, Antalya, 2007.

[27] Durán, M.J., Levi, E., "Multi-Dimensional Space Vector Pulse Width Modulation for Disturbance-Free Operation of a Five-Phase AC Motor Drive”, in Proc. Int. Power Electronics and Motion Control Conf. EPE-PEMC, Aalborg, Denmark, 2007.

[28] H.M. Ryu, J.H. Kim, S.K. Sul, “Analysis of multi-phase space vector pulse width modulation based on multiple d-q spaces concept," in Proceedings $4^{\text {th }}$ Int. Power Electronics and Motion Control Conf. IPEMC, Xian, China, 2004, CD-ROM paper no. 2183.

[29] H.M. Ryu, J.H. Kim, S.K. Sul, “Analysis of multi-phase space vector pulse width modulation based on multiple d-q spaces concept," IEEE Trans. on Power Electronics, vol. 20, no. 6, 2005, 1364-1371. 